\title{
Avascular bone necrosis of the femoral head after renal transplantation: Is it avoidable?
}

\author{
Ayman F. Refaie, M.D. ${ }^{1}$; Zeyad Abd El-Gawad, Ms.C. ${ }^{1}$; Mohamed A. Fouda, M.D. ${ }^{1}$; Ahmed F. Hamdy, M.D. ${ }^{1}$; \\ Mohamed A. Bakr, M.D. ${ }^{1}$; Amani Mostafa, M.D. ${ }^{2}$; Mohamed I. Abo El Ghar, M.D. ${ }^{3}$; Huda F. Refaie, M.D. ${ }^{3}$; \\ Sameh Bahgat, M.D. ${ }^{5}$; El Metwaly El Shahawy, M.D. ${ }^{5}$ and Mohamed A. Ghoneim, M.D. (Hon.), FACS (Hon.) ${ }^{4}$ \\ Departments of Nephrology ${ }^{1}$;mmunology ${ }^{2}$; Radiology ${ }^{3}$ and Urology ${ }^{4}$ - Urology \& Nephrology Center, Mansoura University and \\ Department of Internal Medicine ${ }^{5}$, Benha University, Egypt.
}

\begin{abstract}
Background: Avascular osteonecrosis (AVN) is a serious osseous complication after renal transplantation (RT). Its prevalence clearly decreased from $20 \%$ to $4 \%$ possibly due to the use of calcinurin inhibitors $(\mathrm{CNI})$, reduction of steroid doses and use of steroid free regimens. The aim of our study was to evaluate the frequency of AVN among our kidney transplant recipients and to determine the risk factors for its occurrence.

Patients and methods: Among 1785 kidney transplant recipients who received renal allografts between March 1976 and December 2005, 40 patients (2.24\%) developed AVN with a mean age of $31.3 \pm 10.2$ years. Eighty kidney transplant recipients without AVN were selected to be a matched control group. The localization of AVN was the femoral head in all cases.

Results: AVN was diagnosed at a mean of 20.4 months after transplantation. The following risk factors were statistically significant; sirolimus-based regimen, hypercholesterolemia, overweight with body mass index (BMI)>26 and those with HLA A9, HLA B35 and DR B15.

Conclusions: We concluded that the proper management of hypercholesterolemia, maintenance of ideal body weight as well as avoidance of sirolimus-based immunosuppressive regimen in genetically predisposed patients may be an effective preventive strategy to avoid AVN.
\end{abstract}

Correspondence and offprint requests to: Dr. Ayman F. Refaie, M.D, Consultant Nephrologist, Urology and Nephrology Center, Mansoura University, 35516, Mansoura, Egypt

Tel.: 20-50-2262226

Fax: 20-50-2263717

E-mail: ayman_refaie@yahoo.com

\section{Introduction}

Renal transplantation (RT) is the treatment of choice for most patients with end stage renal disease (ESRD). However, it is attended by complications which are many and varied osseous complications after RT include osteoporotic fractures and osteonecrosis. It has been estimated that osteonecrosis of the femoral head affect up to $20 \%$ of recipients. Symptomatic cases of avascular necrosis (AVN) of femoral head are almost always serious complications, accounting for considerable functional restriction and eventual disability, ultimately leading to femoral collapse and total hip arthroplasty in over $80 \%$ of cases [1]. X-rays are usually normal at the early stage of bone disease, whereas magnetic resonance imaging (MRI) is more sensitive and specific for the diagnosis of AVN [2].

The known risk factors for AVN include high cumulative steroid dose, blood urea nitrogen level, hypofibrinolysis caused by variant plasminogen activator inhibitor-1 genotype and allograft rejection. In children, the risk factors for osteonecrosis are poorly known, although age under 10 years seems to decrease the risk and higher cumulative steroid doses to increase the risk of AVN [3]. If the diagnosis is made in early phase, treatment by bisphosphonate infusion and vascular bone grafting may be beneficial. Secondary deformities and osteoarthritis are treated with corrective osteomies and total joint replacement [4].

We retrospectively reviewed our renal transplant recipients followed between March 1976 and December 2005 to identify the frequency and the risk factors for the occurrence of AVN. 
Material and methods

Between March 1976 and December 2005, 1785 kidney transplantations were performed. Forty cases of symptomatic AVN were diagnosed by X-ray exams, or MRI. The demographic characteristics, clinical features, immunosuppressive protocol, rejection history, cumulative dose of corticosteroids, posttransplantation weight gain, duration on dialysis before RT, original kidney disease, HLA and DR loci and serum creatinine of these patients were compared with 80 matched renal transplant recipients without AVN.

Different strategies for management of AVN either core decompression or hip replacements were also studied.

Statistical analysis: Qualitative data were displayed in cross tabulation and quantitative data were described of arithmetic mean $\pm \mathrm{SD}$. Vicariate techniques were used for initial evaluation of contrast. Thus, the chi-square and fisher exact test were used for comparison of frequencies of qualitative variables and unpaired t test was used for comparison of means of two quantitative variables. $\mathrm{P}$ value $<0.05$ was considered significant. All analyses were carried out using the computer package SPSS for windows, release 10 SPSS Inc Chicago, USA, 1993.

\section{Results}

The 40 patients with AVN of the femoral head included 32 men and 8 women. The mean age was $31.3 \pm 10.2$ years. The demographic characteristics, clinical information and biochemical data of the 40 patients with AVN and the 80 control patients are summarized in table(1). The original kidney disease, duration of dialysis, pretransplant corticosteroid use as well as the body mass index were compared with control group.

Table (2) shows that HLA-A9, B35 and DR-B15 were significantly higher among AVN group compared to that of control. Serum cholesterol was significantly higher in the AVN group at initial evaluation and continued to be higher at time of diagnosis of AVN (table 1,2). ON the other hand, the cumulative dose of steroid and the incidence of acute rejection episode were closely similar in both groups. Body mass index was found to be significantly higher among recipients of AVN $(\mathrm{p}=0.05)$.

It is noteworthy that, the incidence of AVN was significantly higher among patients with sirolimus-based immunosuppressive regimen being $6.5 \%$ compared to those maintained on other regimens $(1.8 \%), \mathrm{p}=0.0001$ (table 3).

Table 1. Demographics and clinical data of AVN and control groups.

\begin{tabular}{llll}
\hline & $\begin{array}{l}\text { AVN group } \\
(n=40)\end{array}$ & $\begin{array}{l}\text { Control group } \\
(n=80)\end{array}$ & $P$-value \\
& & & 0.8 \\
Mean age (years ) & $31.3 \pm 10.2$ & $30.3 \pm 9.4$ & 0.7 \\
Gender (male / female) & $32 / 8$ & $64 / 16$ & 0.7 \\
Original kidney disease & & $12(15 \%)$ & 0.8 \\
$\quad$ - Glomerular & $8(20 \%)$ & $23(28.9 \%)$ & 0.8 \\
$\quad$ - Non glomerular & $9(22.5 \%)$ & $45(56.1 \%)$ & 0.7 \\
$\quad$ - Not identified & $23(57.5 \%)$ & $71(90.6 \%)$ & 0.6 \\
Prior hemodialysis & $34(84.6 \%)$ & $1(1.4 \%)$ & 0.7 \\
Prior peritoneal dialysis & $1(2.9 \%)$ & $8(10 \%)$ & 0.8 \\
Pre- emptive transplantation & $5(12.5 \%)$ & $27.9 \pm 12.5$ & 0.9 \\
Duration of dialysis (months) & $27.8 \pm 12.8$ & $6(7.5 \%)$ & 0.7 \\
Prior corticosteroid therapy & $8(20 \%)$ & $21.3 \pm 2.7$ & 0.01 \\
Duration of transplantation (months) & $21.4 \pm 2.7$ & $22.8 \pm 4.3$ & \\
Body mass index & $22.9 \pm 4.9$ & $151 \pm 52.3$ & \\
Serum cholesterol (mg/ dl) & $111.5 \pm 54.7$ & & \\
& & & \\
\hline
\end{tabular}

Table 2. Evaluation of clinical \& biochemical data of AVN and control group at diagnosis of AVN.

\begin{tabular}{llll}
\hline & $\begin{array}{l}\text { AVN Group } \\
(n=40)\end{array}$ & $\begin{array}{l}\text { Control Group } \\
(n=80)\end{array}$ & $P$-value \\
\hline Acute rejection episode & $0.33 \pm 0.64$ & $0.31 \pm 0.61$ & 0.7 \\
Cumulative mean dose of steroid (gm) & $12.3 \pm 15.3$ & $11.5 \pm 13.7$ & 0.98 \\
DEXA Scan (T score) & $-1.39 \pm 0.6$ & $-1.6 \pm 0.8$ & 0.8 \\
BMI & $26.4 \pm 4.6$ & $24.7 \pm 4.2$ & 0.009 \\
HLA - A 9 & $8(20 \%)$ & $4(5 \%)$ & 0.02 \\
HLA - B 35 & $5(12.5 \%)$ & $2(2.5 \%)$ & 0.04 \\
HLA - DR - B 15 & $7(17.5 \%)$ & $5(6.3 \%)$ & 0.3 \\
Serum creatinine (mg/dl) & $1.59 \pm 0.7$ & $1.45 \pm 0.5$ & 0.01 \\
Serum cholesterol (mg/dl) & $206 \pm 58.9$ & $182 \pm 57.4$ & \\
\hline
\end{tabular}


Table 3. Incidence of AVN relative to primary immunosuppressive regimen.

\begin{tabular}{llc}
\hline $\begin{array}{l}\text { Cyclosporine (CsA)-based } \\
21 / 1162(1.8 \%)\end{array}$ & $\begin{array}{l}\text { Non CsA-based } \\
19 / 623(3.1 \%)\end{array}$ \\
$\begin{array}{ll}\text { Tacrolimus-based } \\
6 / 230(2.6 \%)\end{array}$ & $\begin{array}{l}\text { Non tacrolimus-based } \\
34 / 1555(2.3 \%)\end{array}$ \\
Sirolimus- based & Non sirolimus-based & 0.9 \\
$9 / 137(6.5 \%)$ & $30 / 1648(1.8 \%)$ & 0.0001 \\
& & \\
Steroid-free & Non steroid-free & 0.9 \\
$1 / 72(1.3 \%)$ & $39 / 1713(2.3 \%)$ &
\end{tabular}

Figure 1 shows, T1-waighted coronal magnetic resonance (MR) images of a 37-year old male. He reported hip and groin pain on the left side. The MR images show ostenonecrosis of the femoral head with bone edema on the left side. Another 30-year old male patient experienced bilateral AVN of the femoral heads. Figure 2 show the same above mentioned changes but more on the right side.

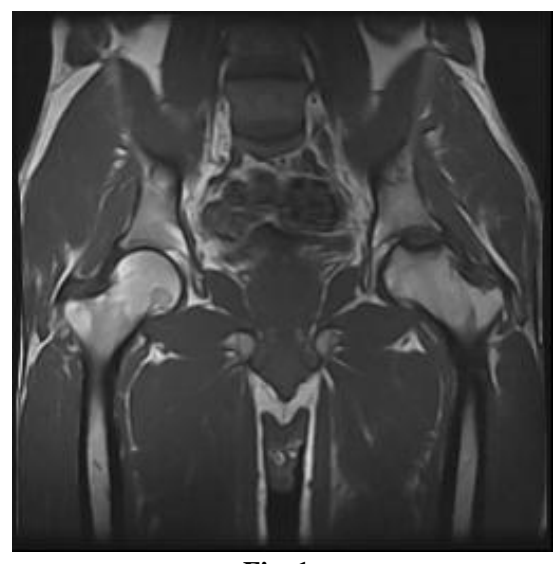

Fig. 1 a

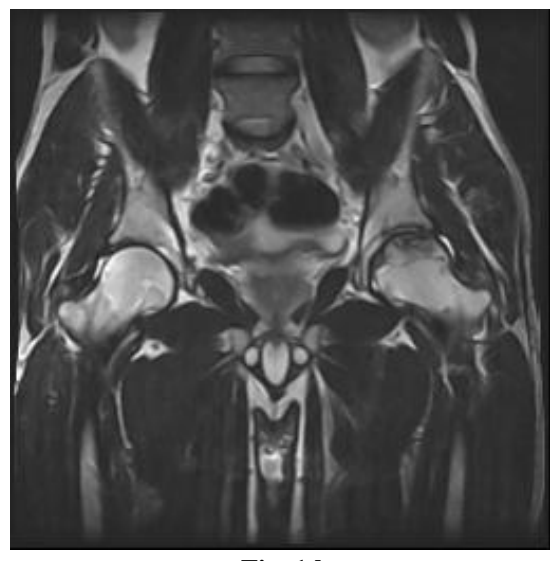

Fig. 1 b

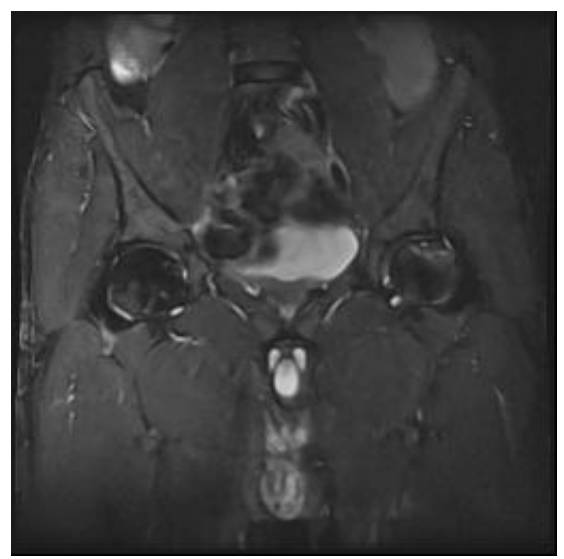

Fig. 1 c

Fig. 1. Unilateral avascular necrosis: The left femoral head showing abnormal subchondrial area of low SI at T1WI (a), high SI at T2WI (b) surrounded by adjacent marrow oedema of high SI at STIR (c).

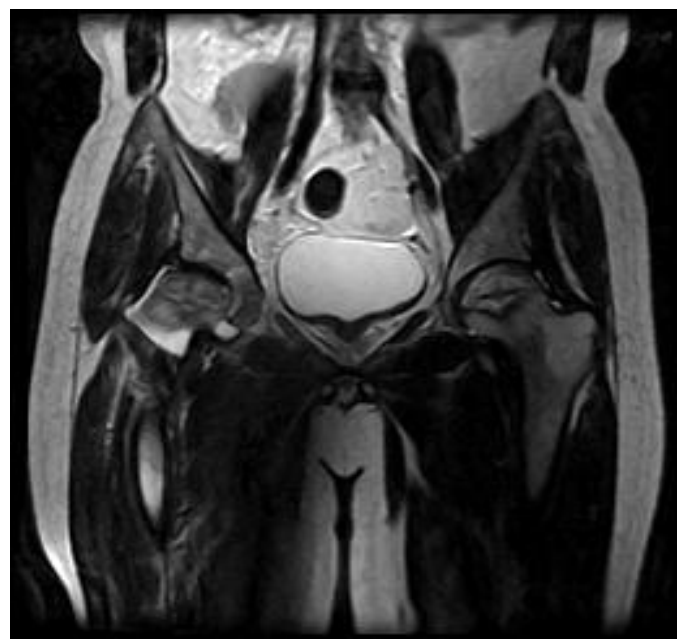

Fig. 2 a

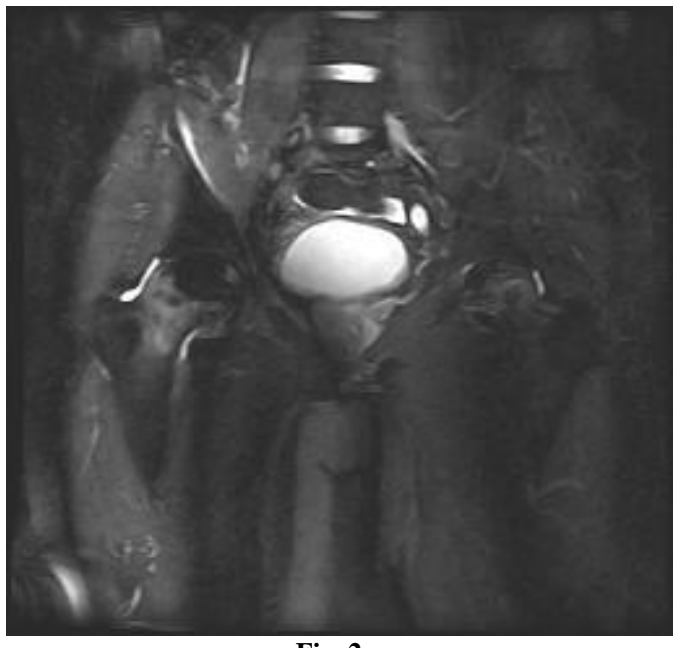

Fig. 2 a

Fig. 2. Bilateral avascular necrosis (AVN) of both femoral heads with mild joint effusion: Both femoral heads are seats of irregular line of granulation tissue surrounding subcortical marrow area of intermediate SI at T2WI (a) and STIR image (b).

\section{Discussion}

Osteoarticular diseases are common in solid organ transplant recipients. The underlying pathophysiology is a complex one. Patients with renal failure have multifactorial bone disease already prior to transplantation. After transplantation, several factors contribute to osteoarticular disease [5]. In the current 
retrospective study, we observed symptomatic AVN in $2.24 \%$. This frequency is probably an underestimate since the screening of AVN was not systematic and imaging exams were performed when patients complained of articular pain evoking AVN. The use of steroid-sparing regimens has dramatically reduced the incidence of AVN from $20 \%$ to no more than $5 \%$ [6]. A review of literatures revealed that the time interval between transplantation and the development of AVN ranges between five and 126 months [7] and it was 20.4 months in our study.

Glucocorticoids are a risk factor for AVN. In $90 \%$ of cases the femoral head is affected. Implantation of a total hip replacement during the further course is often unavoidable in such cases [18]. We found that steroidfree regimen as a primary immunosuppression was not associated with a lower incidence of hip AVN compared with other immunosuppressive protocols and this differs with other reports $[8,9]$ that attributed this to possible alteration in circulating lipids with resultant microemboli in the arteries supplying bone. Another theory proposing that steroids induce changes in venous endothelial cells leading to stasis, increased intraosseous pressure and eventual necrosis [10]. We could attribute our opposing results to the relatively small number of patients on steroid-free regimen. On the other hand, we found no correlation between post transplant AVN and total dose of steroids till diagnosis of AVN or the number of steroid pulses used for acute rejection episodes. Chen and associates [11] reported the same findings and explained these results by the possible genetic predisposition and idiosyncratic response to corticosteroids determined by undefined physiological factors. Moreover, Koo and colleagues [12] found that the total dose of steroid used until AVN was diagnosed by MRI ranged from 1.8 to $15.5 \mathrm{gm}$ of prednisolone or its equivalent. In our results, the cumulative dose of steroids was higher in AVN group (12.7 gm) compared with $11.4 \mathrm{gm}$ in the control group but this did not attain statistical significance.

On focusing on different immunosuppressive regimens, we found a highly significant correlation between post transplant AVN and the use of sirolimus-based regimen (6.5\% versus $1.8 \%$ in non sirolimus-based regimen, $\mathrm{p}<0.0001)$. Bhandari and Eris [12] reported the same findings and explained this by the adverse lipid profile induced by sirolimus, its potent bone marrow suppressive effect or perhaps an idiosyncratic effect.

Also, our results showed significant correlation between post transplant AVN and hypercholesterolemia either before or after transplantation. These results agree with that reported by Moscal et al. [13]. This could be explained by the fact that hypercholesterolemia could lead to subchondral fat emboli and endothelial damage. Therefore, monitoring and treatment of hypercholesterolemia should be an essential target after transplantation.

Tsakiris et al. [14] found that excessive weight gain $(>10 \mathrm{~kg})$ in the first 6 months after RT has been demonstrated to be an additional risk factor for the development of AVN. This coincides with our results which showed that the body mass index (BMI) at time of diagnosis of AVN was significantly higher than that of control group $(\mathrm{P}=0.05)$. Inoue and Ono [15] attributed this to the mechanical overload exerted on the weak weight bearing hip joint or microfractures that can induce changes in the blood supply leading to AVN.

Interestingly, we found predominant loci in the AVN group which are: HLA A9 $(\mathrm{P}=0.009)$, HLA B35 $(\mathrm{P}=0.02)$ and HLA DR B15 $(\mathrm{P}=0.04)$, denoting that patients with these loci could be genetically predisposed to osteonecrosis. The genetic basis for hip AVN was studied by Chen et al. [11] and their results indicated that a 15-cM region between D12S1663 and D12S1632 in chromosome 12 q13 might harbor a gene for AVN of the femoral head.

About $55 \%$ of our patients were managed by core decompression only, $20 \%$ by hip replacement, and $12.5 \%$ by core decompression followed by hip replacement while the remaining were managed conservatively. Core decompression is effective only in early stages. So, the use of MRI in suspected and high risk patients should be adopted after transplantation [5,7].

Conclusion: The incidence of posttransplant AVN of the femoral head was $2.24 \%$ among our cases. The most important risk factors for the development of AVN include; recipients with HLA A9, HLA B35 \& DR B15, BMI > 26: Hypercholesterolemia and sirolimus-based immunosuppressive regimen. Steroid-free regimen deserves further evaluation as it seems a logic tool to lower the incidence of AVN. Finally, kidney transplant recipients should be advised to maintain ideal body weight and it is crucial to monitor and treat hypercholesterolemia. Moreover, it seems wise to adopt steroid and sirolimus-free immunosuppressive regimen for kidney transplant candidates having the above mentioned HLA-DR loci in an attempt to avoid AVN.

\section{References}

1. Lopez-Ben R, Mikuls TR, Moore DS, Julian BA, Bernreuter WK, Elkins M, Saag KG.: Incidence of hip osteonecrosis among renal transplantation recipients: a prospective study. Clin Radiol. 2004; 59(5): 431-438.

2. Hedri H, Cherif M, Zouaghi K, Abderrahim E, Goucha R, Ben Hamida F, Ben Abdallah T, Elyounsi F, Ben Moussa F, Ben Maiz $\mathrm{H}$, Kheder A.: Avascular osteonecrosis after renal transplantation. Transplant Proc. 2007; 39 (4):1036-1038.

3. Helenius I, Jalanko H, Remes V, Tervahartiala P, Salminen S, Sairanen H, Holmberg C, Helenius M, Nietosvaara Y, Peltonen J.: Avascular bone necrosis of the hip joint after solid organ transplantation in childhood: a clinical and MRI analysis. Transplantation. 2006; 81 (12):1621-1627.

4. Helenius I, Remes V, Salminen S, Valta H, Mäkitie O, Holmberg C, Palmu P, Tervahartiala P, Sarna S, Helenius M, Peltonen J, Jalanko H.: Incidence and predictors of fractures in children after solid organ transplantation: a 5-year prospective, populationbased study. J Bone Miner Res. 2006; 21 (3):380-387.

5. Sayed-Noor AS.: Joint arthroplasties other than the hip in solid organ transplant recipients. Open Orthop J. 2009; 15; 3:27-31.

6. Torres A, Lorenzo V, Salido E: Calcium metabolism and skeletal problems after transplantation. J Am Soc Nephrol. 2002; 13 (2): 551-558. 
7. Scott B, Marston MD, Gillingham K, Robert F, Bailey LPN, Edward YC: Osteonecrosis of the femoral head after solid organ transplantation. J Bone Joint Surg Am. 2002; 84(12):2145-2151.

8. Schrem H, Barg-Hock H, Strassburg CP, Schwarz A, Klempnauer J.: Aftercare for patients with transplanted organs. Disch Arztebl Int. 2009; 106(9):148-156.

9. Matas AJ, Kandaswamy R, Gillingham KJ, MeHugh L, Ibrahim $\mathrm{H}$, Kasiske B, Humar A.: Prednisone-free maintenance immunosuppression-a 5-year experience. Am J Transplant. 2005; 5 (10): 2473-2478.

10. Nishimura T, Matsumuto T, Nishino $M$, Tomita $K$ : Histopathologic study of veins in steroid treated rabbits. Clin Orthop Relat Res. 1997; (334):37-42.

11. Chen WM, Liu YF, Lin MW, Chen IC, Lin PY, Lin GL, Jou YS, Lin YT, Fann CS, WU JY, Hsiao KJ, Tsai SF.: Autosomal dominant avascular necrosis of femoral head in two Taiwanese pedigrees and linkage to chromosome 12q13. Am J Hum Genet. 2004; 75 (2) 310-317.

12. Bhandari S, Eris J. Drug points: Premature osteonecrosis and sirolimus treatment in renal transplantation. BMJ 2001; 22; 323 (7314): 665 .

13. Moskal JT, Topping RE, Franklin LL.: Hypercholesterolemia: an association with osteonecrosis of the femoral head. Am J Orthop (Belle Mead NJ) 1997; 26(9):609-612.

14. Tsakiris D, Caslake MJ, Briggs JD, Packard CJ, Shepherd J.: Low-density lipoprotein metabolism following renal transplantation. Transplantation. 1985; 39(4):458-460.

15. Inoue A, Ono K.: A histological study of idiopathic avascular necrosis of the head of the femur. J Bone Joint Surg Br. 1979; 61B (2):138-143. 